\title{
Incidence and risk factors for seizures associated with deep brain stimulation surgery
}

\author{
Travis J. Atchley, MD, ${ }^{1}$ Galal A. Elsayed, MD, ${ }^{1}$ Blake Sowers, BS, ${ }^{2}$ Harrison C. Walker, MD, ${ }^{3}$ \\ Gustavo Chagoya, MD, ${ }^{1}$ Matthew C. Davis, MD, MPH, ${ }^{1}$ Joshua D. Bernstock, MD, PhD, ${ }^{4}$ \\ Nidal B. Omar, MD,' Daxa M. Patel, MD,' and Barton L. Guthrie, MD'
}

Departments of ${ }^{1}$ Neurosurgery and ${ }^{3}$ Neurology, University of Alabama at Birmingham; ${ }^{2}$ University of Alabama at Birmingham School of Medicine, Birmingham, Alabama; and 4Department of Neurological Surgery, Brigham and Women's Hospital, Harvard Medical School, Boston, Massachusetts

OBJECTIVE The objective of this study was to determine the incidence of seizures following deep brain stimulation (DBS) electrode implantation and to evaluate factors associated with postoperative seizures.

METHODS The authors performed a single-center retrospective case-control study. The outcome of interest was seizure associated with DBS implantation. Univariate analyses were performed using the Student t-test for parametric continuous outcomes. The authors used the Kruskal-Wallis test or Wilcoxon rank-sum test for nonparametric continuous outcomes, chi-square statistics for categorical outcomes, and multivariate logistic regression for binomial variables.

RESULTS A total of 814 DBS electrode implantations were performed in 645 patients (478 [58.7\%] in men and 520 [63.9\%] in patients with Parkinson's disease). In total, 22 (3.4\%) patients who had undergone 23 (2.8\%) placements experienced seizure. Of the 23 DBS implantation-related seizures, 21 were new-onset seizures (3.3\% of 645 patients) and 2 were recurrence or worsening of a prior seizure disorder. Among the 23 cases with postimplantation-related seizure, epilepsy developed in 4 (17.4\%) postoperatively; the risk of DBS-associated epilepsy was $0.50 \%$ per DBS electrode placement and $0.63 \%$ per patient. Nine (39.1\%) implantation-related seizures had associated postoperative radiographic abnormalities. Multivariate analyses suggested that age at surgery conferred a modest increased risk for postoperative seizures (OR 1.06, 95\% Cl 1.02-1.10). Sex, primary diagnosis, electrode location and sidedness, and the number of trajectories were not significantly associated with seizures after DBS surgery.

CONCLUSIONS Seizures associated with DBS electrode placement are uncommon, typically occur early within the postoperative period, and seldom lead to epilepsy. This study suggests that patient characteristics, such as age, may play a greater role than perioperative variables in determining seizure risk. Multiinstitutional studies may help better define and mitigate the risk of seizures after DBS surgery.

https://thejns.org/doi/abs/10.3171/2020.5.JNS20125

KEYWORDS seizure; epilepsy; deep brain stimulation; complications; Parkinson's disease; essential tremor; functional neurosurgery

$\mathrm{D}$ EEP brain stimulation (DBS) has become a standard treatment for medically refractory Parkinson's disease, essential tremor, and dystonia and continues to show promise in other neurological and psychiatric disorders. While adverse events are rare, the most common nonhardware complications after DBS surgery include perioperative altered mental status $(1.7 \%-3.5 \%)$, symptomatic intraparenchymal hemorrhage $(0.8 \%-1.1 \%)$, and ischemic infarction $(0.4 \%){ }^{1,2}$ In addition, seizures dur- ing or after DBS surgery have been reported infrequently. Prior studies have reported an incidence ranging from $0.4 \%$ to $4 \% .^{1-6}$ In the majority of patients (up to $100 \%$ in some studies), DBS implantation-associated seizures occur within 48 hours after implantation. ${ }^{1,7,8}$ Given the paucity of relevant studies and the low incidence, seizures associated with DBS surgery require further investigation, given that these events can alter or interrupt DBS surgery and significantly impact quality of life. The objective of

ABBREVIATIONS DBS $=$ deep brain stimulation.

SUBMITTED January 13, 2020. ACCEPTED May 11, 2020.

INCLUDE WHEN CITING Published online August 7, 2020; DOI: 10.3171/2020.5.JNS20125. 
this study was to determine the incidence and potential risk factors for seizures during or immediately after DBS surgery.

\section{Methods \\ Ethics}

This paper was prepared in accordance with the Strengthening the Reporting of Observational Studies in Epidemiology (STROBE) guidelines. The University of Alabama at Birmingham IRB approved this study.

\section{Study Design}

This is a retrospective case-control study on all patients who underwent DBS electrode implantation at a tertiary care university center. Variables of interest included sex, diagnosis, presence of preoperative seizure disorder, age at surgery, hemisphere implanted, unilateral versus bilateral implantation, number of trajectories, intra- and/or postoperative seizures, radiographic findings in those with implantation-associated seizures, development of postimplantation seizure disorder, and length of postoperative follow-up.

\section{Data Collection}

All adults (defined as age $>18$ years) who underwent DBS electrode implantation from April 1998 to January 2019 with at least 1 month of follow-up were included in the analysis. The outcome of interest was identified by querying diagnoses of "seizure" and/or "epilepsy" via ICD-9 and/or ICD-10 diagnosis codes. Electronic medical records were reviewed for all patients with any of the relevant ICD-9 or ICD-10 codes to determine the nature, chronicity, and postoperative features associated with seizures. Seizure was defined as an applicable ICD-9 or ICD10 code with any of the following documented in the medical record: abnormal electroencephalography findings, transient focal deficit without imaging findings to suggest a separate etiology, witnessed seizure, or unwitnessed seizure event with a documented postictal state. For the purposes of this study, epilepsy or DBS implantation-associated seizure disorder were defined as seizures $>24$ hours apart or an inability to wean off antiepileptic medication according to follow-up documentation. Patients with a preexisting seizure disorder were considered to have an implantation-associated seizure if, in the postoperative period, they had recurrence of otherwise controlled seizures, had increased frequency of their usual seizure, or had a clinically different seizure semiology than usual. The electronic medical record was extensively reviewed to ensure that these patients had an objective and/or quantifiable change in their known seizure disorder prior to inclusion in the DBS surgery-associated seizure group.

\section{Statistical Analysis}

Descriptive statistics were generated on demographic data using core functions in $\mathrm{R}$ in the RStudio environment. We confirmed normality where appropriate and utilized parametric and nonparametric techniques for univariate analysis where appropriate. We used the Kruskal-Wallis test and the Wilcoxon rank-sum test for nonparametric univariate statistical analyses associated with continuous dependent variables, and the chi-square test was used for categorical continuous dependent variables. Power analyses were performed with a two-sided test for the Wilcoxon rank-sum test, with an alpha of 0.05 , a normal distribution, and a large effect size (0.8). Empirical power was demonstrated at near 1 for both continuous and categorical outcomes. Multivariate logistic regression was performed on binomial outcome variables with respect to covariates and postimplantation epilepsy. These covariates included sex, diagnosis, age at surgery, history of seizure disorder, hemisphere implanted, unilateral versus bilateral implantation, and number of trajectories.

\section{Results}

\section{Patient Demographics and DBS Implantation-Associated Seizure Incidence}

A total of 814 DBS electrode implantations were performed in 645 patients. Table 1 shows patient demographics and seizure incidence. Male patients accounted for 478 (58.7\%) DBS procedures, and 520 (63.9\%) electrodes were implanted for Parkinson's disease. The median duration of postoperative follow-up was 50 months (IQR 7-71 months). Patients in $8(0.98 \%)$ cases had a preexisting seizure disorder. Table 2 details the characteristics of patients with DBS implantation-related seizures. Seizures occurred after 23 (2.8\%) electrode implantations (22 [3.4\%] unique patients). Of the 806 electrode placements (637 patients without a preexisting seizure disorder), 21 (2.6\%) procedures (20 [3.1\%] patients) were associated with newonset seizures. Postoperative seizures in 14 (63.6\%) cases occurred less than 24 hours after placement (median postoperative day 1, IQR 0-3 days). Postimplantation epilepsy occurred in $4(17.4 \%)$ of the 23 seizure cases, and 2 $(8.70 \%)$ had worsening or return of a preexisting seizure disorder. Excluding the patients in 8 cases with a prior seizure disorder, new-onset DBS electrode implantation-associated epilepsy occurred in $4(0.50 \%)$ of 806 electrode placements (4 [0.63\%] of 637 patients). Radiographic abnormalities ranging from mild edema to intraparenchymal hemorrhage with mass effect were seen in $9(39.1 \%)$ of the 23 cases of electrode placement-associated seizures. Of the 4 patients with new-onset DBS electrode placementassociated epilepsy, 2 had focal electrode edema and 1 had a small tract hemorrhage on postoperative imaging.

\section{Univariate Analysis}

Table 3 shows results of the univariate analysis. Preexisting seizure disorder was significantly associated with postoperative seizures $(\mathrm{p}<0.001)$ only in this univariate model. Sex, primary diagnosis, electrode location/sidedness, age at surgery, and number of trajectories were not significantly associated with the onset of new seizures.

\section{Multivariate Analysis}

Results of the multivariate analysis are shown in Table 4. Age at surgery was significantly associated with elevated risk for postimplantation seizures (OR 1.06, 95\% CI 1.02-1.10). Preexisting seizure disorder was not sig- 
TABLE 1. Demographics of 645 patients who underwent 814 electrode implantations for DBS

\begin{tabular}{lc}
\hline & Value \\
\hline Male sex & $478(58.7)$ \\
\hline Primary diagnosis & $520(63.9)$ \\
\hline Parkinson's disease & $221(27.1)$ \\
\hline Essential tremor & $49(6.0)$ \\
\hline Dystonia & $24(2.9)$ \\
\hline Other & \\
\hline Electrode target & $407(50)$ \\
\hline STN & $276(33.9)$ \\
\hline VIM & $131(16.1)$ \\
\hline GPi & $497(61.1)$ \\
\hline Lt-sided electrode & $63.8 \pm 11.4(63.8)$ \\
\hline Mean age at op \pm SD (median), yrs & $1.48 \pm 0.73$ \\
\hline Mean no. of trajectories \pm SD & $563(69.2)$ \\
\hline Laterality & $208(25.6)$ \\
\hline Initial placement & $43(5.3)$ \\
\hline 2nd side or initially bilateral implant & $23(2.8)$ \\
\hline Other* & $8(0.98)$ \\
\hline DBS surgery-associated seizure &
\end{tabular}

$\mathrm{GPi}=$ globus pallidus internus; $\mathrm{STN}$ = subthalamic nucleus; $\mathrm{VIM}=$ ventral intermediate nucleus.

Values are presented as the number of procedures (\%) unless stated otherwise.

* Includes DBS lead replacement after removal of infected/malfunctioning, eroded, or malpositioned hardware.

nificantly associated with postimplantation recurrence or exacerbation of seizures (OR $0.02,95 \% \mathrm{CI}-1.87$ to 3.92 ).

\section{Discussion}

Seizures related to DBS surgery are uncommon and usually do not result in epilepsy. Other common adverse events of device failure, infection, and erosion of hardware are well described in the literature. ${ }^{2,9}$ However, the postimplantation seizure incidence is less well defined, occurring at a reported rate of $0.4 \%-4 \% .{ }^{1-6}$ In one of the largest single-institution patient populations, DBS surgery-associated seizures occurred after $23(2.8 \%)$ DBS placement procedures (3.3\% of patients), consistent with prior studies. Most seizures $(60.9 \%)$ resolved within 24 hours with a median follow-up of 4 months.

Our study includes 645 patients who underwent 814 DBS electrode implantation procedures from 1998 to 2019. This is one of the largest single-institution studies with some of the longest patient follow-up found in the literature. New-onset seizures occurred in $2.6 \%$ of procedures (3.1\% of patients). Few studies have looked specifically at the incidence of electrode placement-associated epilepsy. We found that new-onset post-DBS placement epilepsy occurred after $0.5 \%$ of implantation procedures and in $0.63 \%$ of patients. Importantly, we did not find that postDBS seizures acted as harbingers for subsequent infection, at least within 3 months postoperatively. We also noted
TABLE 2. Demographics of patients with DBS electrode implantation-associated seizure

\begin{tabular}{cc}
\hline \multicolumn{2}{c}{ Value } \\
\hline Electrode target \\
\hline STN & $16(69.6)$ \\
\hline VIM & $5(21.7)$ \\
\hline GPi & $2(8.7)$ \\
\hline Lt-sided electrode & $18(78.3)$ \\
\hline Mean postop day of seizure \pm SD & $2.8 \pm 4.9$ \\
\hline Median postop day of seizure (IQR) & $1(0-3)$ \\
\hline Postop radiographic abnormality & $14(60.9)$ \\
\hline None & $5(21.7)$ \\
\hline Hemorrhage & $4(17.4)$ \\
\hline Electrode-associated edema & $14(63.6)$ \\
\hline Resolution of seizures & $8(36.4)$ \\
\hline$<24$ hrs & $1(4.3)$ \\
\hline$>24$ hrs & $50(7-71)$ \\
\hline Missing
\end{tabular}

Values are presented as the number of cases (\%) unless stated otherwise.

TABLE 3. Univariate analysis of patients with and without DBS electrode implantation-associated seizure

\begin{tabular}{|c|c|c|c|}
\hline & No Seizure & Seizure & $\mathrm{p}$ Value \\
\hline No. of procedures & 791 & 23 & \\
\hline Sex & & & 0.133 \\
\hline Male & $461(58.3)$ & $17(73.9)$ & \\
\hline Female & $330(41.7)$ & $6(26.1)$ & \\
\hline Preexisting seizure disorder & $6(0.8)$ & $2(8.7)$ & $<0.001$ \\
\hline Diagnosis & & & 0.708 \\
\hline Parkinson's disease & $504(63.7)$ & $16(69.6)$ & \\
\hline Other & $287(36.4)$ & $6(26.1)$ & \\
\hline Electrode target & & & 0.161 \\
\hline STN & $391(49.4)$ & $16(69.6)$ & \\
\hline VIM & $271(34.3)$ & $5(21.7)$ & \\
\hline GPi & $129(16.3)$ & $2(8.7)$ & \\
\hline Electrode sidedness & & & 0.218 \\
\hline Rt & $312(39.4)$ & $5(21.7)$ & \\
\hline $\mathrm{Lt}$ & $479(60.6)$ & $18(78.3)$ & \\
\hline Mean age at op $\pm S D$, yrs & $63.9 \pm 11.3$ & $59.0 \pm 13.5$ & 0.138 \\
\hline Stage of DBS & & & 0.687 \\
\hline Unilateral & $536(69.4)$ & $15(65.2)$ & \\
\hline $\begin{array}{l}\text { 2nd side or initially bilateral } \\
\text { implant }\end{array}$ & $199(25.8)$ & $6(26.1)$ & \\
\hline Other & $37(4.8)$ & $2(8.7)$ & \\
\hline Missing & $19(2.4)$ & 0 & \\
\hline Mean no. of trajectories \pm SD & $1.46 \pm 0.67$ & $1.70 \pm 1.02$ & 0.374 \\
\hline
\end{tabular}

Values are presented as the number of procedures (\%) unless stated otherwise. Boldface type indicates statistical significance. 
TABLE 4. Multivariate logistic regression for DBS surgeryassociated seizure risk factors

\begin{tabular}{lll}
\hline & \multicolumn{1}{c}{ OR $(95 \% \mathrm{Cl})$} & $\mathrm{p} \mathrm{Value}$ \\
\hline Female sex & $2.19(1.12$ to 3.27$)$ & 0.152 \\
\hline Essential tremor diagnosis & $1.97(0.62$ to 3.32$)$ & 0.324 \\
\hline Dystonia diagnosis & $0.88(-0.79$ to 2.55$)$ & 0.882 \\
\hline Other diagnosis & $6.43(2.81$ to 10.05$)$ & 0.314 \\
\hline Age at op & $1.06(1.02$ to 1.10$)$ & 0.008 \\
\hline Lt hemispheric implant & $0.38(-0.73$ to 1.48$)$ & 0.085 \\
\hline 2nd stage implant placement & $0.63(-0.45$ to 1.70$)$ & 0.395 \\
\hline 1st stage or other implant & $0.43(-1.22$ to 2.08$)$ & 0.315 \\
\hline No. of trajectories & $0.72(0.19$ to 1.24$)$ & 0.208 \\
\hline Preexisting seizure disorder & $0.02(-1.87$ to 3.92$)$ & 0.027 \\
\hline
\end{tabular}

Boldface type indicates statistical significance.

postimplantation hemorrhagic radiographic abnormalities (subdural and/or intraparenchymal hemorrhage) after 5 (21.7\%) electrode placements. In a previous study, we found that $18.8 \%$ of patients had pneumocephalus and $7.6 \%$ had hemorrhagic complications. ${ }^{2}$ While we did not specifically investigate postoperative imaging for nonseizure patients, both Nazzaro et al. and Seijo et al. have previously reported this increased risk of seizure associated with abnormal imaging findings. ${ }^{10,11}$ This is one of the few studies to investigate epilepsy after DBS surgery given the low incidence of seizure reported in the literature. In this large series, we looked at multiple intraoperative features and found no association with perioperative seizure.

Preexisting seizure disorder was not found to be associated with postimplantation seizures in multivariable analyses. We hypothesized that even patients with controlled seizures preoperatively were at risk for postoperative epileptic activity, but this increased risk was only demonstrated on univariate analysis. However, the number of patients with preexisting seizure disorder with a presumed secondary increase in seizure postimplantation is small $(n=2)$, and the analysis may be underpowered to determine an actual increased odds of worsening seizure. Both of the patients with preexisting seizure disorder who developed new seizures after DBS surgery were compliant with valproic acid prior to surgery. Our local practice is to continue home antiepileptic medications prior to DBS surgery, and we do not initiate any additional seizure prophylaxis in this higher-risk population. We found a modest association between younger age and postimplantation seizures (OR 1.06). While this increased risk is small, it may represent a decreased tolerance for edema or hemorrhage within younger brains. Concurrently, with a presumed greater volume of brain matter, pneumocephalus may result in greater cortical irritation in younger patients. In contrast, however, Pouratian et al. found that age $>60$ years was associated with seizures in univariate analysis, but this observation did not reach significance in a subsequent multivariable model. ${ }^{8}$

Seijo et al. reported seizures in 3.15\% of DBS procedures and similar resolution within 12 hours postoperatively. ${ }^{11}$ A systematic review by Coley et al. reported an overall post-DBS seizure risk of $2.4 \%(1.7 \%-3.3 \%)$ and an estimated delayed or nonperioperative seizure incidence of $0.5 \%(0.02 \%-1.00 \%)]^{7}$ Beric et al. investigated DBS surgery complications in 86 patients and reported on 2 patients with intraoperative tonic-clonic seizures, one of whom subsequently developed epilepsy. ${ }^{12}$ Multiple other studies have corroborated the spontaneous resolution of seizures postoperatively, with nearly all patients experiencing seizure freedom within 6 months. ${ }^{7,8,11,13}$ These data suggest that the majority of seizures may be related to transient cortical irritation that resolves spontaneously.

In a previous study, we determined that postoperative imaging abnormalities (pneumocephalus, hemorrhage, or edema) were not associated with seizure incidence. ${ }^{2}$ However, others have demonstrated an association between postoperative imaging abnormalities and seizures. Wang et al. found that $50 \%$ of patients with postoperative intraparenchymal hemorrhage had associated seizures. ${ }^{14}$ Nazzaro et al. reported seizures as the presenting symptom in all patients with delayed lead-associated edema. ${ }^{10}$ Similarly, Seijo et al. found that abnormal postoperative imaging findings (intracranial hemorrhage, edema, or ischemia) were associated with seizure development (OR 50.4, 95\% CI 5.7-444.3).$^{11}$ Boviatsis et al. reported generalized tonic-clonic seizures following bilateral DBS placement with pneumocephalus demonstrated on postoperative imaging. 15

A transventricular electrode trajectory has been reported in association with postimplantation seizures. ${ }^{8}$ However, the current study and that of Seijo et al. did not find any association between the number of trajectories and seizure incidence. ${ }^{5}$ While the majority of DBS electrodes were targeted to the subthalamic nucleus, the anatomical target of the DBS electrode was not significantly associated with postoperative seizures in the present study.

\section{Limitations}

This study carries the inherent limitations of a retrospective chart review study. Data were collected from a retrospective database and are subject to risks of improper or inadequate documentation. We relied on our clinical database, ICD codes, and the electronic medical record to determine the incidence of postoperative seizures, and some patients may have been missed if they were not given the designated ICD codes. While we report on a large number of procedures, de novo seizures are infrequent adverse events, and determining the associated risk factors is difficult with small numbers of the outcome of interest. A multiinstitutional collaboration could help overcome this limitation. Imaging findings were not collected in all patients, only in those with DBS implantation-associated seizures. Therefore, the association between radiographic abnormalities and seizure onset was not reported in this study. However, Patel et al. performed a more detailed imaging review at our institution. ${ }^{2}$ Given the limitations of available data and the number of models in the multivariable model, there is the potential for overfitting of the data, which may skew our final results. Finally, some patients were lost to follow-up, and there was a large degree of variation among length of follow-up duration. Therefore, the prevalence of delayed postoperative seizures and/or epilepsy may be misrepresented. 


\section{Conclusions}

DBS implantation-associated seizure is an uncommon occurrence, with an overall observed risk of $2.8 \%$ per procedure. The majority of these events occurred within the early postoperative period (within 48 hours). Newonset seizures occurred with $2.6 \%$ of procedures $3.1 \%$ of patients). Postimplantation DBS epilepsy is an even less common complication, occurring after $0.50 \%$ of DBS procedures and in $0.63 \%$ of patients. Age at surgery was significantly associated with risk of seizure, while number of trajectories, DBS target location, and preexisting seizure disorder were not. These findings suggest that intraoperative parameters may not play as great a role in the risk of DBS implantation-associated seizure as baseline patient characteristics. Multiinstitutional studies may be helpful in stratifying risk for seizures related to DBS implantation.

\section{Acknowledgments}

G.A.E. completed this work as a Clinical Buchalter Scholar.

\section{References}

1. Fenoy AJ, Simpson RK Jr. Risks of common complications in deep brain stimulation surgery: management and avoidance. J Neurosurg. 2014;120(1):132-139.

2. Patel DM, Walker HC, Brooks R, et al. Adverse events associated with deep brain stimulation for movement disorders: analysis of 510 consecutive cases. Neurosurgery. 2015; 11(suppl 2):190-199.

3. Fernández-Pajarín G, Sesar A, Ares B, et al. Delayed complications of deep brain stimulation: 16-year experience in 249 patients. Acta Neurochir (Wien). 2017;159(9):1713-1719.

4. Kenney C, Simpson R, Hunter C, et al. Short-term and longterm safety of deep brain stimulation in the treatment of movement disorders. J Neurosurg. 2007;106(4):621-625.

5. Seijo FJ, Alvarez-Vega MA, Gutierrez JC, et al. Complications in subthalamic nucleus stimulation surgery for treatment of Parkinson's disease. Review of 272 procedures. Acta Neurochir(Wien). 2007;149(9):867-876.

6. Voges J, Hilker R, Bötzel K, et al. Thirty days complication rate following surgery performed for deep-brain-stimulation. Mov Disord. 2007;22(10):1486-1489.

7. Coley E, Farhadi R, Lewis S, Whittle IR. The incidence of seizures following deep brain stimulating electrode implantation for movement disorders, pain and psychiatric conditions. Br J Neurosurg. 2009;23(2):179-183.

8. Pouratian N, Reames DL, Frysinger R, Elias WJ. Comprehensive analysis of risk factors for seizures after deep brain stimulation surgery. J Neurosurg. 2011;115(2):310-315.

9. Atchley TJ, Laskay NMB, Sherrod BA, et al. Reoperation for device infection and erosion following deep brain stimulation implantable pulse generator placement. J Neurosurg. 2020;133(2):403-410.
10. Nazzaro JM, Pahwa R, Lyons KE. Symptomatic, non-infectious, non-hemorrhagic edema after subthalamic nucleus deep brain stimulation surgery for Parkinson's disease. $J$ Neurol Sci. 2017;383:42-46.

11. Seijo F, Alvarez de Eulate Beramendi S, Santamarta Liébana E, et al. Surgical adverse events of deep brain stimulation in the subthalamic nucleus of patients with Parkinson's disease. The learning curve and the pitfalls. Acta Neurochir (Wien). 2014;156(8):1505-1512.

12. Beric A, Kelly PJ, Rezai A, et al. Complications of deep brain stimulation surgery. Stereotact Funct Neurosurg. 2001; 77(1-4):73-78.

13. Sorar M, Hanalioglu S, Kocer B, et al. Experience reduces surgical and hardware-related complications of deep brain stimulation surgery: a single-center study of 181 patients operated in six years. Parkinsons Dis. 2018;2018:3056018.

14. Wang X, Wang J, Zhao H, et al. Clinical analysis and treatment of symptomatic intracranial hemorrhage after deep brain stimulation surgery. Br J Neurosurg. 2017;31(2):217222.

15. Boviatsis EJ, Stavrinou LC, Themistocleous M, et al. Surgical and hardware complications of deep brain stimulation. A seven-year experience and review of the literature. Acta Neurochir (Wien). 2010;152(12):2053-2062.

\section{Disclosures}

Dr. Walker: consultant for Medtronic and Boston Scientific and research funding from the National Institutes of Health (UH3 NS100553) and the Michael J. Fox Foundation (MJFF 15098). Dr. Bernstock: positions/equity in CITC Ltd. and Avidea Technologies and member of the board of scientific advisors for POCKiT Diagnostics.

\section{Author Contributions}

Conception and design: Guthrie, Atchley, Sowers, Davis. Acquisition of data: Atchley, Sowers, Omar, Patel. Analysis and interpretation of data: Atchley, Elsayed, Walker, Chagoya, Bernstock. Drafting the article: Atchley, Elsayed, Sowers, Chagoya. Critically revising the article: Guthrie, Atchley, Elsayed, Walker, Chagoya, Davis, Bernstock, Patel. Reviewed submitted version of manuscript: Guthrie, Atchley, Elsayed, Sowers, Walker, Chagoya, Davis, Bernstock, Omar. Approved the final version of the manuscript on behalf of all authors: Guthrie. Statistical analysis: Atchley, Elsayed. Study supervision: Guthrie.

\section{Correspondence}

Barton L. Guthrie: University of Alabama at Birmingham, AL. bguthrie@uabmc.edu. 